\title{
Serodiagnosis of aspergillosis in falcons (Falco spp.) by Afmp1p-based enzyme-linked immunosorbent assay
}

Chi-Ching Tsang $^{a, *}$, Ulrich Wernery ${ }^{b, *},{ }^{*}$ Christiana Hebelc, Alexandra Dameraud, Jörg Kinne $^{b}$, Jian-Piao Caia, Harald Küsperte, Ka-Fai Chana, Marina Joseph ${ }^{b}$, Shaolong Xue $^{a}$, Rekha Raghavan ${ }^{b}$, James Y. M. Tanga, Ginu Syriac ${ }^{b}$, Susanna K. P. Lau, ${ }^{a, f, g, h, i}$,

ISHAM Shantymol Jose ${ }^{b}$ and Patrick C. Y. Woo ${ }^{a, f, g, h, i}$

aDepartment of Microbiology, Li Ka Shing Faculty of Medicine, The University of Hong Kong, Hong Kong; bentral Veterinary Research Laboratory, Dubai, UAE; 'F3 Falcons R, Dubai, UAE; dUniversity of Applied Sciences, Berlin, Germany; eFalcon Center GbR, Helvesiek, Germany; fResearch Centre of Infection and Immunology, The University of Hong Kong, Hong Kong; gState Key Laboratory of Emerging Infectious Diseases, The University of Hong Kong, Hong Kong; hCarol Yu Centre for Infection, The University of Hong Kong, Hong Kong; and 'Collaborative Innovation Centre for Diagnosis and Treatment of Infectious Diseases, The University of Hong Kong, Hong Kong

\section{Introduction}

- Aspergillosis has emerged as one of the main health problems in raptors (e.g. falcons)

- Falcons with aspergillosis have a high mortality rate

- Diagnostic methods include endoscopy, mycological culture and cytology/histology, etc.

- Afmp1p is a cell wall galactomannoprotein of Aspergillus fumigatus, which is highly antigenic

- Anti-Afmp1p antibodies were detected from sera of human aspergillosis patients by Afmp1p-based ELISA $\rightarrow$ Potential use for diagnosing human cases

- Hypothesis: Could this Afmp1p-based ELISA be used for serodiagnosis of aspergillosis in falcons?

\section{Methodology}

- Generation and characterisation of a guinea pig polyclonal HRP-conjugated anti-falcon IgY

- Experimental infection with A. fumigatus

- Three peregrine falcons (Falco peregrinus), one as negative control

- Four saker (F. cherrug) x gyrfalcons (F. rusticolus), two as negative controls

- Detection of immunoreaction between recombinant ( $r$ )-Afmp1p and sera from experimentally infected falcons

- ELISAs

- rAfmp1p as coating antigen

- Characterisation of antibody response of the experimentally infected falcons

- Evaluation of the anti-Afmp1p-based ELISA system using serum samples from captive-bred falcons (12 with and 129 without aspergillosis) collected in a clinic in Dubai, UAE

\section{Results}

$\boldsymbol{\nabla}$ Titre and detection limit of anti-falcon IgY

Titre $\left(E C_{50}\right)=1: 7,332 \quad$ Detection limit $=2,538 \mathrm{pg} / \mathrm{mL}$
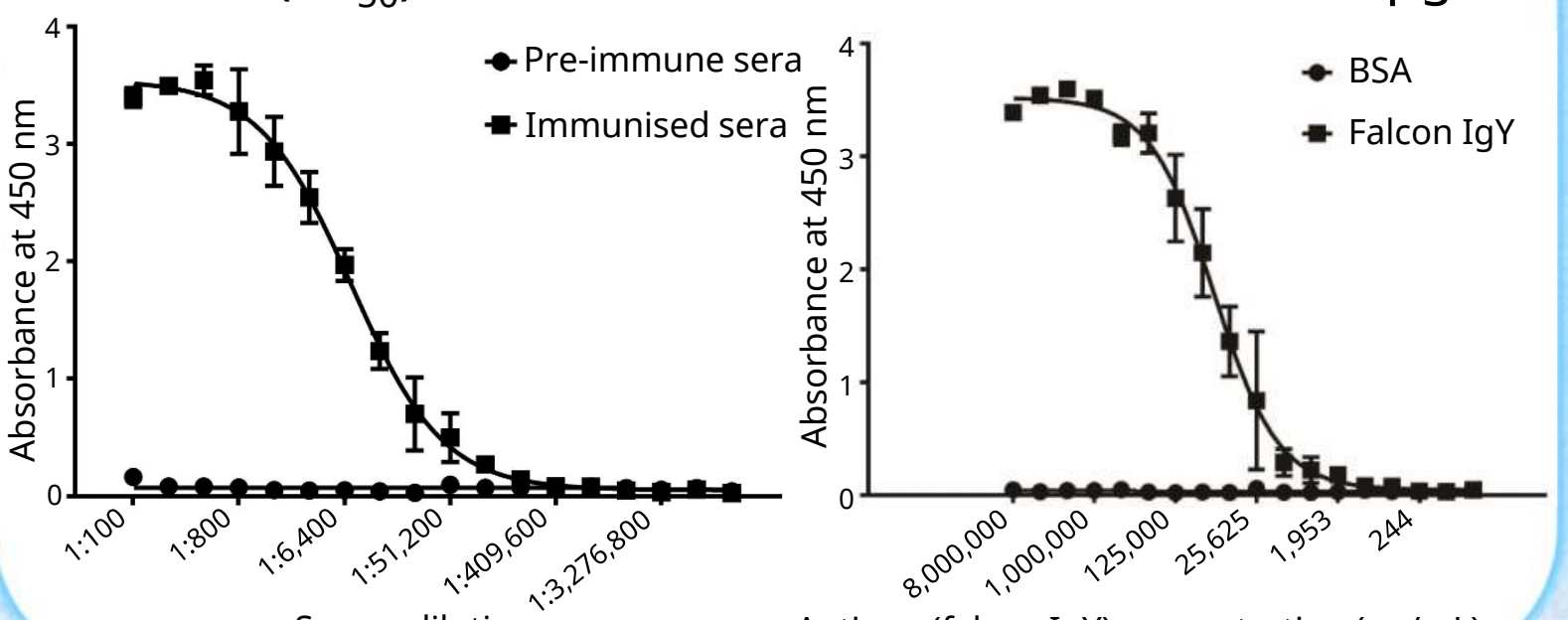

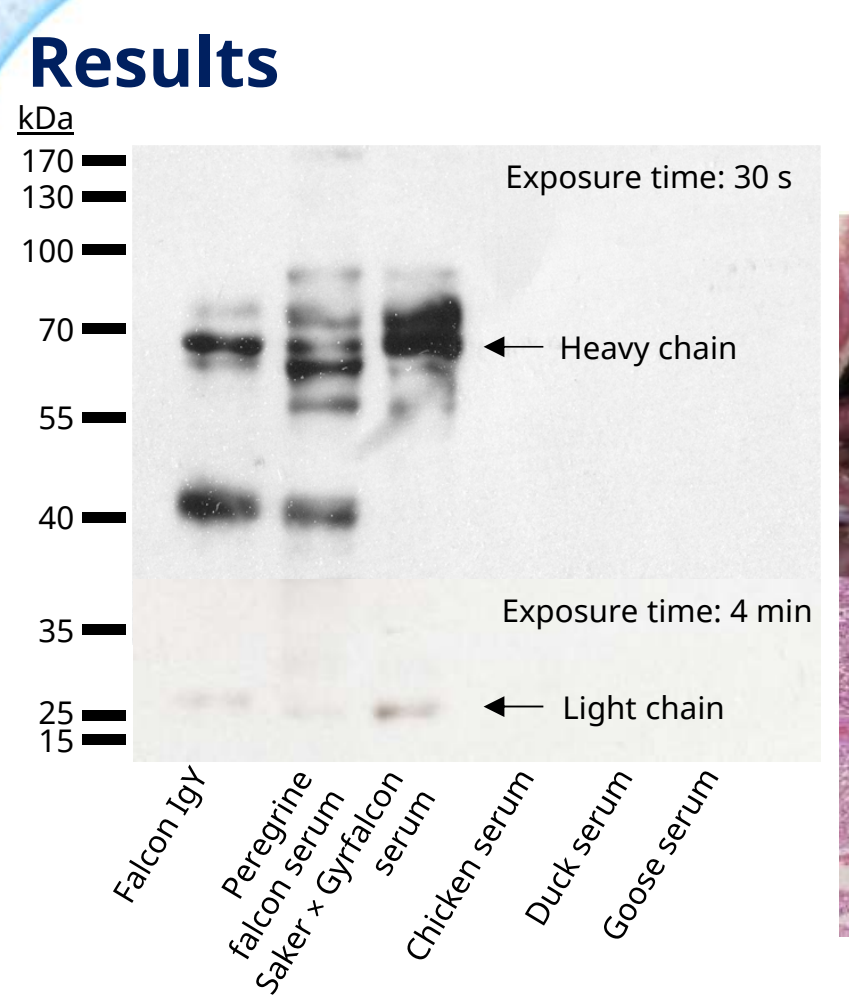

$\boldsymbol{\nabla}$ Necropsy and histology of an experimentally infected falcon

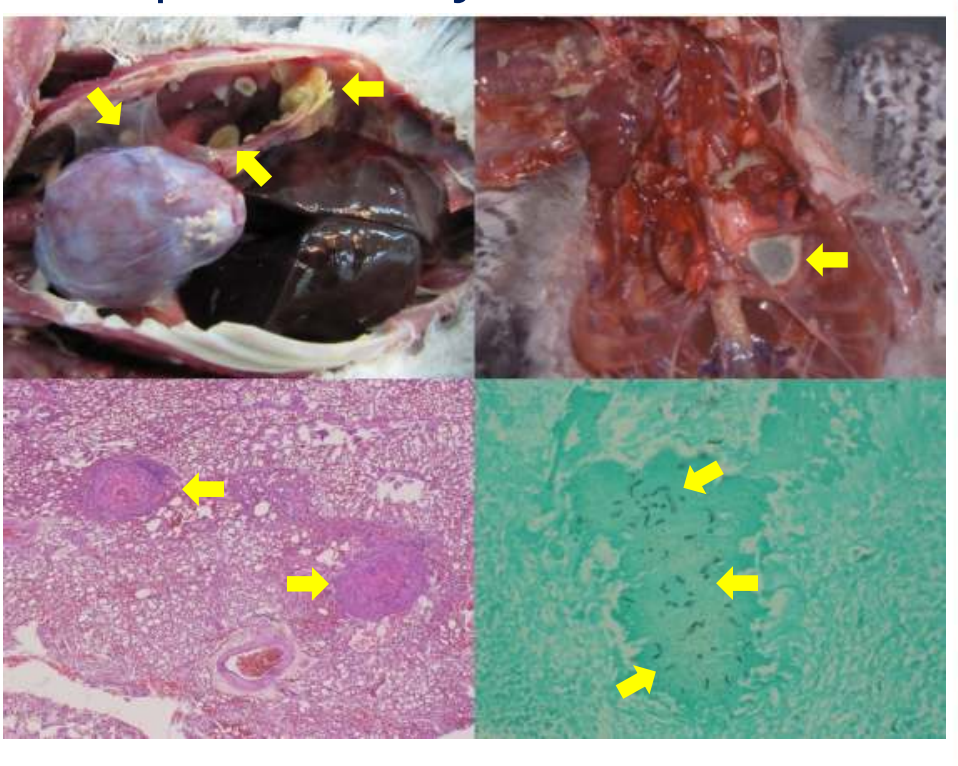

$\Delta$ Anti-falcon IgY is specific to falcons

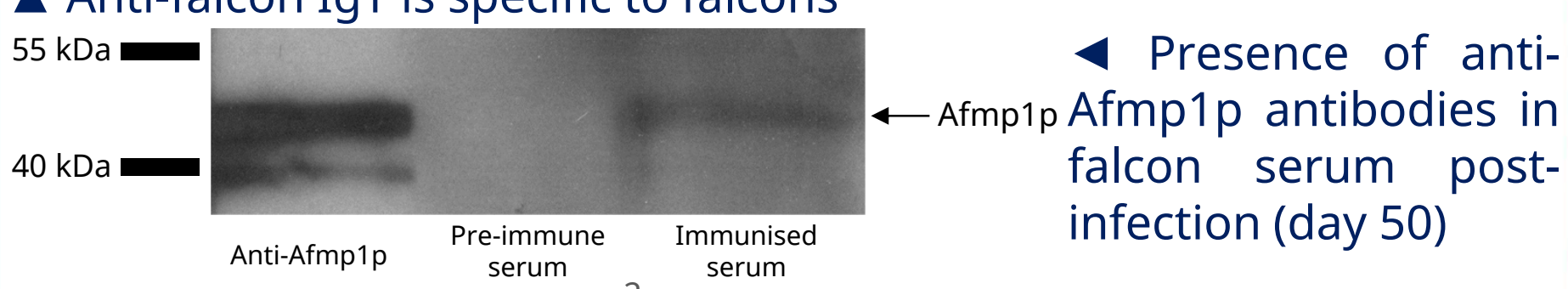

- Serum anti-Afmp1p 䲶 1.8 antibody profiles of 1.6 the experimentally infected falcons

$(P$, peregrine falcons; SG, saker $\times$ gyrfalcons: P3, SG3 \& SG4 are uninfected negative controls)
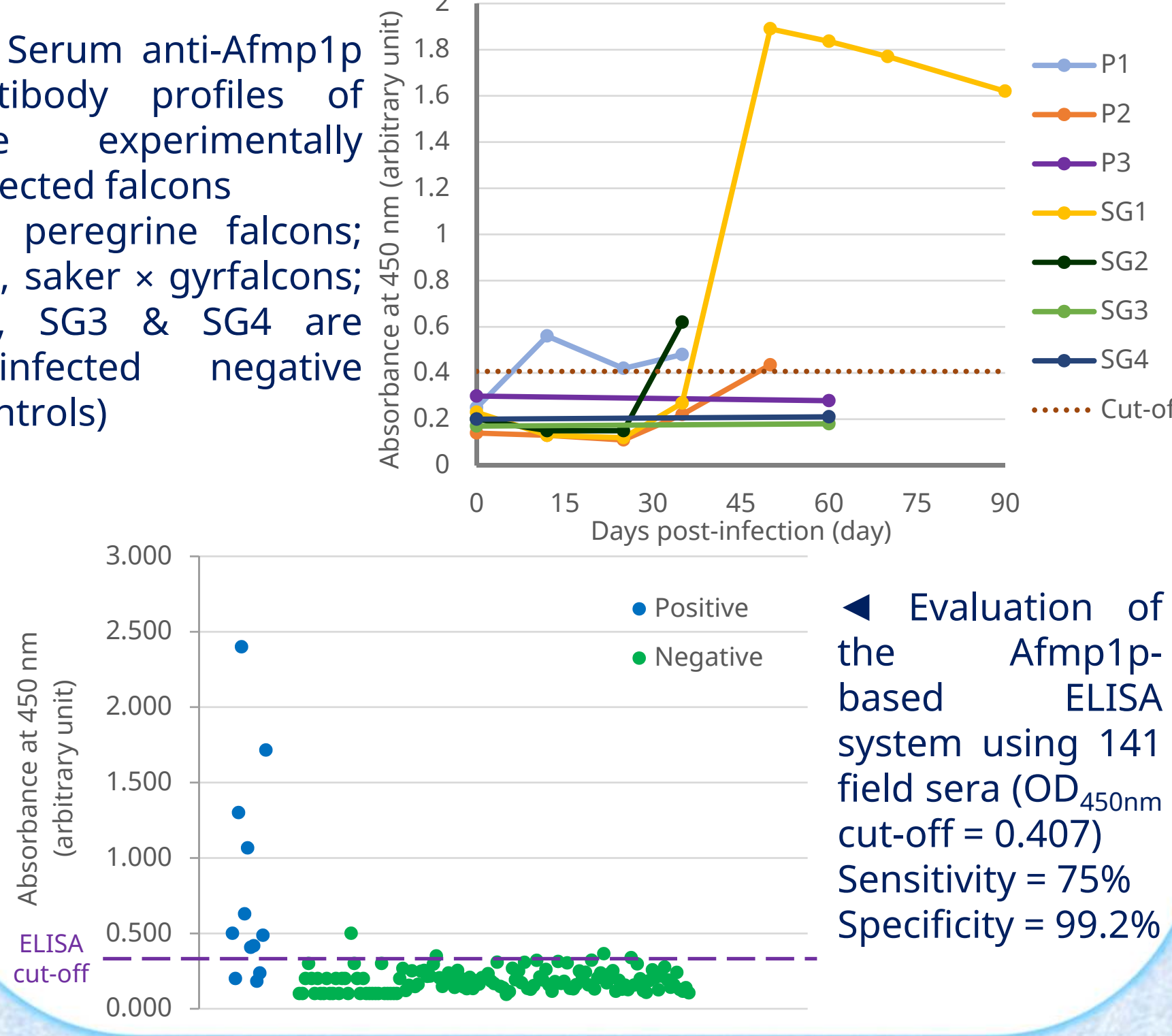

\section{Acknowledgements}

WE WHE UNIVERSITY OF HONG KONG

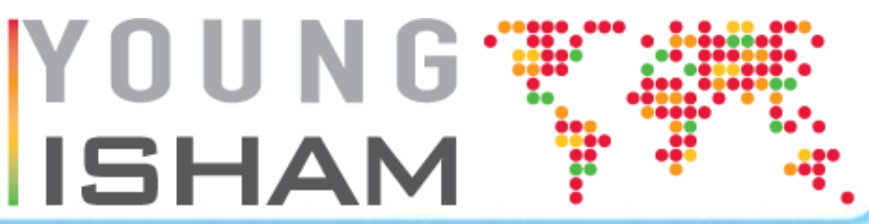

\section{Reference}

